\title{
ON THE TYPES OF LINEAR PARTIAL DIFFERENTIAL EQUATIONS OF THE SECOND ORDER IN THREE INDEPENDENT VARIABLES WHICH ARE UNALTERED BY THE TRANSFORMATIONS OF A CONTINUOUS GROUP*
} BY

\section{J. E. CAMPBELL}

\section{Introduction.}

It is known (Messenger of Mathematics, November, 1898, p. 97) that the equation :

$$
\frac{\partial^{2} z}{\partial x_{1}^{2}}+\frac{\partial^{2} z}{\partial x_{2}^{2}}+\frac{\partial^{2} z}{\partial x_{3}^{2}}=0
$$

is unaltered by any infinitesimal transformation of a certain group of the tenth order, and that all such transformations which leave the equation unaltered are contained in the above group. It is at once evident that any equation which by a point transformation can be reduced to the above form will also be unaltered by the transformations of a group of the tenth order of like composition with the above. In the present paper a more general proposition is considered, viz., the form to which linear partial differential equations, of the second order in three independent variables, can be reduced which have the property of being unaltered for some infinitesimal transformations. Such equations form a class by themselves, the potential equation above and equations reducible to it by point transformation being only particular types of this class; it is here shown that the infinitesimal transformations which leave unaltered the equations of this class form in all cases a finite group of the eleventh order at highest; and certain types are tabulated to which all equations of the class may be reduced.

\section{$\S 1$. The definition equations of the group.}

Let $x_{1}, x_{2}, x_{3}$ be three independent variables, $z$ the dependent variable, and let the linear partial differential equation of the second order be written :

$$
\begin{aligned}
a_{11} z_{11} & +a_{22} z_{22}+a_{33} z_{33}+2 a_{23} z_{23}+2 a_{31} z_{31}+2 a_{12} z_{12} \\
& +2 a_{1} z_{1}+2 a_{2} z_{2}+2 a_{3} z_{3}+a z=0 ;
\end{aligned}
$$

* Presented to the Society, December 28, 1899. Received for publication, December 29, 1899. 
it is required to find the conditions that the equation may be unaltered by the infinitesimal transformation which consists in substituting for $x_{i}, z$,

$$
\begin{array}{ll}
x_{i}+\xi_{i}\left(x_{1}, x_{2}, x_{3}\right) t^{*} & (i=1,2,3), \\
z+z \zeta\left(x_{1}, x_{2}, x_{3}\right) t, &
\end{array}
$$

respectively, where $t$ is a constant so small that its square may be neglected; in other words the conditions are required that the equation (1) may admit the infinitesimal transformation $X$ where

$$
X \equiv \xi_{1} \frac{\partial}{\partial x_{1}}+\xi_{2} \frac{\partial}{\partial x_{2}}+\xi_{3} \frac{\partial}{\partial x_{3}}+z \zeta \frac{\partial}{\partial z} .
$$

By a method almost identical with that given in the Proceedings of the London Mathematical Society, vol. 29, p. 251, we see that by this transformation $z_{i}$ becomes $z_{i}+\pi_{i} t$ and $z_{i \kappa}$ becomes $z_{i \kappa}+\pi_{i \kappa} t$, where

$$
\begin{gathered}
\pi_{i}=\zeta z_{i}+z \frac{\partial \zeta}{\partial x_{i}}-\sum_{\kappa=1}^{\kappa=3} z_{\kappa} \frac{\partial \xi_{\kappa}}{\partial x_{i}}, \\
\pi_{i \kappa}=z_{i \kappa} \zeta+z_{i} \frac{\partial \zeta}{\partial x_{\kappa}}+z_{\kappa} \frac{\partial \zeta}{\partial x_{i}}+z \frac{\partial^{2} \zeta}{\partial x_{i} \partial x_{\kappa}}-\sum_{r=1}^{r=3} z_{\kappa r} \frac{\partial \xi_{r}}{\partial x_{i}}-\sum_{r=1}^{r=3} z_{i r} \frac{\partial \xi_{r}}{\partial x_{\kappa}}-\sum_{r=1}^{r=3} z_{r} \frac{\partial^{2} \xi_{r}}{\partial x_{i} \partial x_{\kappa}} \\
\quad(i, \kappa=1,2,3) .
\end{gathered}
$$

The condition that (1) is unaltered implies that

$$
\begin{gathered}
\sum_{i, \kappa} a_{i \kappa} \pi_{i \kappa}+2 \sum_{i} a_{i} \pi_{i}+a z \zeta \\
+(\rho-\zeta)\left(\sum_{i, \kappa} a_{i \kappa} z_{i \kappa}+2 \sum_{i} a_{i} z_{i}+a z\right) \\
+\sum_{i, \kappa}\left(X a_{i \kappa}\right) z_{i \kappa}+2 \sum_{i}\left(X a_{i}\right) z_{i}+(X a) z \equiv 0,
\end{gathered}
$$

where $i, \kappa=1,2,3$ and $\rho$ is some undetermined function.

* Lre proves (Leipziger Berich te, 1894-95, p. 322) that a contact transformation which transforms every linear partial differential equation into a linear one of the same order must be of the form $x_{1}^{\prime}=f_{1}\left(x_{1}, x_{2}, x_{3}\right), x_{2}^{\prime}=f_{2}\left(x_{1}, x_{2}, x_{3}\right), x_{3}^{\prime}=f_{3}\left(x_{1}, x_{2}, x_{3}\right), z^{\prime}=z f\left(x_{1}, x_{2}, x_{3}\right)$; and although it does not follow that some one particular linear equation may not have a more general transformation formula conserving its linearity, yet it may easily be verified by a method given in LIE-ENGEL (vol. II, p. 252 and 895 ) that no partial equation of the form $a_{11} z_{11}+a_{22} z_{22}+$ $a_{33} z_{33}$ (and to such a form the equations here considered may be reduced) can conserve its linearity for an infinitesimal contact transformation unless the characteristic function $w$ is of the form:

$$
z_{1} f_{1}\left(x_{1}, x_{2}, x_{3}\right)+z_{2} f_{2}\left(x_{1}, x_{2}, x_{3}\right)+z_{3} f_{3}\left(x_{1}, x_{2}, x_{3}\right)-z f\left(x_{1}, x_{2}, x_{3}\right)-\phi\left(x_{1}, x_{2}, x_{3}\right) .
$$

It follows that the infinitesimal transformations here considered are essentially of the most general form. See also Crelle's Journal, vol. 114, pp. 116-142; for this reference the author is indebted to Dr. C. L. Bouton. 
Substituting for $\pi_{i \kappa}$ and $\pi_{i}$ and equating coefficients of $z$ and its derivatives we get six equations of the form :

(2) $\quad a_{\kappa 1} \frac{\partial \xi_{i}}{\partial x_{1}}+a_{\kappa 2} \frac{\partial \xi_{i}}{\partial x_{2}}+a_{\kappa 3} \frac{\partial \xi_{i}}{\partial x_{3}}+a_{i 1} \frac{\partial \xi_{\kappa}}{\partial x_{1}}+a_{i 2} \frac{\partial \xi_{\kappa}}{\partial x_{2}}+a_{i 3} \frac{\partial \xi_{\kappa}}{\partial x_{3}}=\rho a_{i \kappa}+X a_{i \kappa}$,

these being obtained by equating the coefficients of the derivatives of the second order; and

$$
\begin{aligned}
\left(\sum_{i, \kappa} a_{i \kappa} \frac{\partial^{2}}{\partial x_{i} \partial x_{\kappa}}\right. & \left.+2 \sum_{i} a_{i} \frac{\partial}{\partial x_{i}}\right) \xi_{1} \\
& -2\left(a_{11} \frac{\partial \zeta}{\partial x_{1}}+a_{12} \frac{\partial \zeta}{\partial x_{2}}+a_{13} \frac{\partial \zeta}{\partial x_{3}}\right) \equiv 2 \rho a_{1}+2 X a_{1}
\end{aligned}
$$

and two other similar equations obtained from derivatives of the first order; and finally

$$
\left(\sum_{i, \kappa} a_{i \kappa} \frac{\partial^{2}}{\partial x_{i} \partial x_{\kappa}}+2 \sum_{i} \dot{a}_{i} \frac{\partial}{\partial x_{i}}\right) \zeta \equiv-\rho a-X a,
$$

obtained by equating the coefficients of $z$.

If values of $\xi_{1}, \xi_{2}, \xi_{3}, \zeta$ can be found to satisfy these ten equations then

$$
\xi_{1} \frac{\partial}{\partial x_{1}}+\xi_{2} \frac{\partial}{\partial x_{2}}+\xi_{3} \frac{\partial}{\partial x_{3}}+z \zeta \frac{\partial}{\partial z}
$$

is a transformation which the equation (1) admits. Now all such transformations obviously form a continuous group, since the operation equivalent to two such successive operations is also an operation which leaves the equation (1) unaltered; it is now to be proved that the group is a finite one.

\section{§ 2. Proof that the group is finite.}

In order to prove this the more readily it will be convenient to transform the equation (1) to one in which the coefficients of $z_{23}, z_{31}, z_{12}$ shall be zero. First it should be stated that in this paper attention is confined to those equations of the form (1) for which the determinant,

$$
\left|\begin{array}{lll}
a_{11} & a_{12} & a_{13} \\
a_{21} & a_{22} & a_{23} \\
a_{31} & a_{32} & a_{33}
\end{array}\right|
$$

which we call $M$ does not vanish. Now for any transformation of the type:

$$
\begin{array}{ll}
x_{i}^{\prime} & =f_{i}\left(x_{1}, x_{2}, x_{3}\right) \\
z^{\prime} & =z f\left(x_{1}, x_{2}, x_{3}\right),
\end{array} \quad(i=1,2,3),
$$


where $f_{1}, f_{2}, f_{3}, f$ are any holomorphic functions, the equation (1) is transformed to another of the same type, the new coefficients being given by equations of the form :

It easily follows that

$$
a_{12}^{\prime} f=\sum_{i, \kappa} a_{i \kappa} \frac{\partial x_{1}^{\prime}}{\partial x_{i}} \frac{\partial x_{2}^{\prime}}{\partial x_{\kappa}} .
$$

$$
M^{\prime} f^{3}=M\left(\frac{\partial\left(x_{1}^{\prime}, x_{2}^{\prime}, x_{3}^{\prime}\right)}{\partial\left(x_{1}, x_{2}, x_{3}\right)}\right)^{2}
$$

so that $M^{\prime} \neq 0$ if $M \neq 0$.

Choosing $x_{1}^{\prime}, x_{2}^{\prime}, x_{3}^{\prime}$ so that $a_{23}^{\prime}=a_{31}^{\prime}=a_{12}^{\prime}=0$, we see that the conditions (2), in order that the transformed (1) may admit

$$
\xi_{1}^{\prime} \frac{\partial}{\partial x_{1}^{\prime}}+\xi_{2}^{\prime} \frac{\partial}{\partial x_{2}^{\prime}}+\xi_{3}^{\prime} \frac{\partial}{\partial x_{3}^{\prime}}+\zeta^{\prime} z^{\prime} \frac{\partial}{\partial z}
$$

take the simpler form (the accents being dropped) :

$$
\left\{\begin{array}{c}
2 a_{i i} \frac{\partial \xi_{i}}{\partial x_{i}}=\rho a_{i i}+X a_{i i} \\
a_{\kappa \kappa} \frac{\partial \xi_{i}}{\partial x_{\kappa}}+a_{i i} \frac{\partial \xi_{\kappa}}{\partial x_{i}}=0
\end{array}\right.
$$

And since the determinant in the original equation does not vanish none of the quantities $a_{11}, a_{22}, a_{33}$ can be zero.

We can now prove that the equation system $(2),(3),(4)$ if consistent involves only a finite number of constants in its solution. To prove this it is only necessary to show that all derivatives can be expressed in terms of a finite number of derivatives of $\xi_{1}, \xi_{2}, \xi_{3}$. (See LIE-ENGEL, Transformationsgruppen, vol. I, chap. X.)

For the sake of conciseness write $\xi, \eta, \zeta$ instead of $\xi_{1}, \xi_{2}, \xi_{3}$ and let $\xi_{i \kappa}$ denote $\partial^{2} \xi / \partial x_{i} \partial x_{\kappa}$ with other similar abbreviations; also write $a, b, c$ for $a_{11}$, $a_{22}, a_{33}$.

We conclude from (5) that

$$
\xi_{1}-\eta_{2}, \quad \eta_{2}-\zeta_{3}, \quad a \zeta_{1}+c \xi_{3}, \quad b \zeta_{2}+c \eta_{3}, \quad a \eta_{1}+b \xi_{2}
$$

can be expressed in terms of $\xi, \eta$ and $\zeta$.

It follows by differentiation that

$$
\xi_{111}-\eta_{112}, \quad \eta_{112}-\zeta_{113}, \quad a \zeta_{113}+c \xi_{133}, \quad \xi_{133}-\eta_{233}
$$

can each be expressed in terms of derivatives of order not exceeding the second, and, therefore, $a \eta_{112}+c \eta_{233}$ can be so expressed. 
Again, $\xi_{122}-\eta_{222}, \eta_{222}-\zeta_{223}, b \zeta_{223}+c \eta_{233}, a \eta_{112}+b \xi_{122}$ can be expressed in terms of derivatives of order not exceeding the second, and, therefore, $a \eta_{112}-c \eta_{223}$ can be so expressed.

We have at once the facts that $\eta_{112}$ and $\eta_{223}$ can each be expressed in terms of derivatives of order two at the most, and immediately deduce that

$$
\eta_{112}, \quad \xi_{111}, \quad \zeta_{113}, \quad \eta_{233}, \quad \xi_{133}, \quad \zeta_{223}, \quad \eta_{222}, \quad \xi_{122}
$$

can each be so expressed.

Once more $a \eta_{13}+b \xi_{23}, a \zeta_{12}+c \xi_{23}, b \zeta_{12}+c \eta_{13}$ can each be expressed in terms of derivatives of order not exceeding one, and, therefore, so can $\xi_{23}, \eta_{31}, \zeta_{12}$; it follows that

$$
\begin{array}{lll}
\xi_{231}, & \eta_{131}, & \zeta_{121}, \\
\xi_{232}, & \eta_{132}, & \zeta_{122}, \\
\xi_{233}, & \eta_{133}, & \zeta_{123}
\end{array}
$$

can each be expressed in terms of derivatives of order not exceeding two.

We can now see that all derivatives of the third order can be expressed in terms of derivatives of lower order ; thus since $\eta_{123}$ and $\xi_{113}-\eta_{123}$ can be so expressed, $\xi_{113}$ can be ; similarly $\zeta_{133}$ can be, and, therefore, since $a \zeta_{133}+c \xi_{333}$ can be, $\xi_{333}$ can also ; $\zeta_{123}$ can be and also $\eta_{122}-\xi_{123}$, therefore $\eta_{122}$; from the facts that $\xi_{112}-\eta_{122}$ and $a \eta_{122}+b \xi_{222}$ can be, we then deduce that $\xi_{112}$ and $\xi_{222}$ can be.

We have now proved that all derivatives of the third order of $\xi$ can be expressed in terms of lower derivatives, and similarly we may prove the proposition for $\eta$ and $\zeta$.

By similar reasoning we may see that there are fifteen linearly independent equations connecting derivatives of the second order, and since there are five equations connecting derivatives of the first order we conclude that all derivatives can be expressed in terms of $\xi, \eta, \zeta$-four derivatives of the first order and three derivatives of the second order.

Returning now to the equations (5) we see that since $a_{11}$ is not zero we can without altering the equation take it a constant so that $X a_{11}=0$; we have then $\rho=2 \partial \xi_{1} / \partial x_{1}$.

If we now write down the equations. which correspond to (3) we see that all derivatives of $\zeta$ can be expressed in terms of derivatives of $\xi_{1}, \xi_{2}, \xi_{3}$, so that we have finally the result that all derivatives of $\xi_{1}, \xi_{2}, \xi_{3}$, and $\zeta$ can be expressed in terms of $\xi_{1}, \xi_{2}, \xi_{3}, \zeta$-four derivatives of the first order and three of the second.

It follows that the values of $\xi_{1}, \xi_{2}, \xi_{3}, \zeta$ which satisfy the equations (if such values exist) cannot contain more than eleven arbitrary constants so that the group is of the eleventh order at highest. 
It is of course not true that a perfectly general linear partial differential equation admits an infinitesimal transformation at all; what we have proved is that no equation with non-vanishing determinant, whatever its form may be, can admit more than eleven independent transformations. We shall now consider in detail the forms to which such equations can be reduced by point transformations if they admit groups of orders one, two or three. Since every infinitesimal transformation of a group with more than three parameters is contained in at least one group of the third order (LIE, vol. I, p. 593), we shall then have the forms to which all equations of form (1) can be reduced if they admit any group whatever.

§ 3. Equations which admit one infinitesimal transformation.

Any linear operator :

becomes

$$
X \equiv \xi_{1} \frac{\partial}{\partial x_{1}}+\xi_{2} \frac{\partial}{\partial x_{2}}+\xi_{3} \frac{\partial}{\partial x_{3}}+\zeta z \frac{\partial}{\partial z}
$$

$$
X\left(x_{1}^{\prime}\right) \frac{\partial}{\partial x_{1}^{\prime}}+X\left(x_{2}^{\prime}\right) \frac{\partial}{\partial x_{2}^{\prime}}+X\left(x_{3}^{\prime}\right) \frac{\partial}{\partial x_{3}^{\prime}}+X\left(z^{\prime}\right) \frac{\partial}{\partial z^{\prime}}
$$

when we apply the transformation :

$$
\begin{aligned}
x_{i}^{\prime} & =f_{i}\left(x_{1}, x_{2}, x_{3}\right), \\
z^{\prime} & =z f\left(x_{1}, x_{2}, x_{3}\right) .
\end{aligned}
$$

Let now $f_{2}, f_{3}$ be any two independent solutions of

$$
\xi_{1} \frac{\partial U}{\partial x_{1}}+\xi_{2} \frac{\partial U}{\partial x_{2}}+\xi_{3} \frac{\partial U}{\partial x_{3}}=0
$$

and let $f_{1}, f$ respectively be solutions of

$$
\begin{aligned}
& \xi_{1} \frac{\partial U}{\partial x_{1}}+\xi_{2} \frac{\partial U}{\partial x_{2}}+\xi_{3} \frac{\partial U}{\partial x_{2}}=1, \\
& \xi_{1} \frac{\partial U}{\partial x_{1}}+\xi_{2} \frac{\partial U}{\partial x_{2}}+\xi_{3} \frac{\partial U}{\partial x_{3}}+\zeta U=0
\end{aligned}
$$

we see that $X$ becomes $\partial / \partial x_{1}^{\prime}$.

It follows that any equation which admits $X$ can by a transformation of the form (7) be reduced to an equation which admits $\partial / \partial x_{1}^{\prime}$.

It is therefore only necessary to find what conditions the coefficients $a_{i \kappa}, a_{i}$ and $a$ must satisfy if the corresponding equation admits $\partial / \partial x_{1}$. 
Putting $\xi_{1}=1, \xi_{2}=\xi_{3}=\zeta=0$ in (2), (3), (4), we get

$$
\begin{aligned}
\frac{\partial}{\partial x_{1}} a_{i \kappa}+\rho a_{i \kappa} & =0 & (i, \kappa=1,2,3) \\
\frac{\partial}{\partial x_{1}} a_{i}+\rho a_{i} & =0 & (i=1,2,3) \\
\frac{\partial}{\partial x_{1}} a+\rho a & =0 . &
\end{aligned}
$$

Now without any loss of generality we can take some one of the coefficients, say $a_{i \kappa}$, to be a constant other than zero (this merely comes to dividing the equation by $a_{i \kappa}$ ), and therefore $X a_{i \kappa}=0$, and consequently $\rho=0$. It follows that all of the coefficients must have the property of being independent of $x_{1}$. Our first result is then that every linear equation which admits any infinitesimal transformation whatever can be reduced to a form in which all the coefficients depend only on $x_{2}$ and $x_{3}$, and conversely any such equation will admit at least one transformation.

\section{§ 4. Equations admitting groups of the second order.}

Let us now consider the form to which equations can be reduced which admit a group of order two. We know (LiE, vol. III, p. 713) that we can take $X_{1}$ and $X_{2}$ to be the infinitesimal transformations of such group where $X_{1}$ and $X_{2}$ are two linear operators such that either $X_{1} X_{2}-X_{2} X_{1}=0$, or $X_{1} X_{2}-X_{2} X_{1}=X_{1}$.

Consider first the case where $X_{1}$ and $X_{2}$ are commutative. Let $X_{1}$ be reduced to the form $\partial / \partial x_{1}$, then the most general linear operator of the form :

$$
\xi_{1} \frac{\partial}{\partial x_{1}}+\xi_{2} \frac{\partial}{\partial x_{2}}+\xi_{3} \frac{\partial}{\partial x_{3}}+\zeta z \frac{\partial}{\partial z}
$$

which is commutative with $\partial / \partial x_{1}$ is obtained by making $\xi_{1}, \xi_{2}, \xi_{3}, \zeta$ depend only on $x_{2}, x_{3}$. Now $X_{1}$ is unaltered by any transformation :

$x_{1}^{\prime}=x_{1}+f_{1}\left(x_{2}, x_{3}\right), \quad x_{2}^{\prime}=f_{2}\left(x_{2}, x_{3}\right), \quad x_{3}^{\prime}=f_{3}\left(x_{2}, x_{3}\right), \quad z^{\prime}=z f\left(x_{2}, x_{3}\right)$.

Apply such a transformation and $X_{2}$ becomes

$$
\begin{aligned}
&\left(\xi_{1}+\xi_{2} \frac{\partial f_{1}}{\partial x_{2}}+\xi_{3} \frac{\partial f_{1}}{\partial x_{3}}\right) \frac{\partial}{\partial x_{1}^{\prime}}+\left(\xi_{2} \frac{\partial f_{2}}{\partial x_{2}}+\xi_{3} \frac{\partial f_{2}}{\partial x_{3}}\right) \frac{\partial}{\partial x_{2}^{\prime}} \\
&+\left(\xi_{2} \frac{\partial f_{3}}{\partial x_{2}}+\xi_{3} \frac{\partial f_{3}}{\partial x_{3}}\right) \frac{\partial}{\partial x_{3}^{\prime}}+\left(\xi_{2} \frac{\partial f}{\partial x_{2}}+\xi_{3} \frac{\partial f}{\partial x_{3}}+\zeta f\right) \frac{z^{\prime}}{f} \frac{\partial}{\partial z^{\prime}}
\end{aligned}
$$

If $\xi_{2}$ and $\xi_{3}$ are both zero, take $f_{2}=\xi_{1}$, and the operator $X_{2}$ takes the form :

$$
x_{2}^{\prime} \frac{\partial}{\partial x_{1}^{\prime}}+\zeta z^{\prime} \frac{\partial}{\partial z^{\prime}} \text {. }
$$


If $\xi_{2}$ and $\xi_{3}$ are not both zero we can choose $f_{3}$ and $f$ so that the coefficients of $\partial / \partial x_{3}^{\prime}$ and $\partial / \partial z^{\prime}$ are zero; $f_{2}$ so that the coefficient of $\partial / \partial x_{2}^{\prime}$ is unity; and $f_{1}$ so that the coefficient of $\partial_{/}^{\prime} \partial x_{1}^{\prime}$ is zero. We see then that the two operators take one of the forms :

$$
\begin{gathered}
X_{1} \equiv \frac{\partial}{\partial x_{1}}, \quad X_{2} \equiv \frac{\partial}{\partial x_{2}} ; \\
X_{1} \equiv \frac{\partial}{\partial x_{1}}, \quad X_{2} \equiv x_{2} \frac{\partial}{\partial x_{1}}+\zeta z \frac{\partial}{\partial z} .
\end{gathered}
$$

We can now prove that an equation with non-vanishing determinant cannot admit a group of the latter form.

Since the equation (1) admits $X_{1}$ we have seen that the coefficients do not involve $x_{1}$; if the equation (1) also admits $X_{2}$ we must be able to satisfy the equations (2), (3), (4) by taking $\xi_{1}=x_{2}, \xi_{2}=0=\xi_{3}=0$. Now $X a_{i k}=0$ (since $a_{i \mathrm{k}}$ does not involve $x_{1}$ ); therefore $\rho a_{22}=\rho a_{33}=0=\rho a_{23}$; if $\rho \neq 0$, it follows that $a_{22}=a_{33}=a_{23}=0$, which would make the determinant zero: if we take $\rho=0$, from the first of equations (2) we get $a_{12}=0$, from the fifth $a_{32}=0$, and from the sixth $a_{22}=0$; which also makes the determinant zero.

The only permutable group of the second order then which can be admitted is the one which can be reduced to the form $X_{1} \equiv \partial / \partial x_{1} X_{2} \equiv \partial / \partial x_{2}$; and we see as before that all the coefficients must now be independent both of $x_{1}$ and $x_{2}$.

Similar reasoning will show us that the only types of infinitesimal operators of the group whose composition is $\left[X_{1} X_{2}\right]=X_{1}$ are

$$
\begin{aligned}
& X_{1} \equiv \frac{\partial}{\partial x_{1}}, \quad X_{2} \equiv x_{1} \frac{\partial}{\partial x_{1}}+x_{2} \frac{\partial}{\partial x_{2}} ; \\
& X_{1} \equiv \frac{\partial}{\partial x_{1}}, \quad X_{2} \equiv x_{1} \frac{\partial}{\partial x_{1}}+\zeta z \frac{\partial}{\partial z}
\end{aligned}
$$

where $\zeta$ depends only on $x_{2}$ and $x_{3}$; and by exactly the same kind of reasoning we see that the latter type is inadmissible.

On taking now $\xi_{1}=x_{1}, \xi_{2}=x_{2}, \xi_{3}=0, \zeta=0$, the equations (2), (3), (4) become :

$$
\begin{aligned}
\rho a_{33}+X a_{33} & =0, & & (\rho-1) a_{13}+X a_{13}=0, \\
(\rho-1) a_{23}+X a_{23} & =0, & & (\rho-2) a_{11}+X a_{11}=0, \\
(\rho-2) a_{22}+X a_{22} & =0, & & (\rho-2) a_{12}+X a_{12}=0, \\
(\rho-1) a_{1}+X a_{1} & =0, & & (\rho-1) a_{2}+X a_{2}=0, \\
\rho a_{3}+X a_{3} & =0, & \rho a+X a & =0 .
\end{aligned}
$$

Here, since all the coefficients are free from $x_{1}$, we may write $x_{2} \partial / \partial x_{2}$ for $X$, and unless $a_{11}=a_{22}=a_{12}=0$ (a case to be excluded since it makes the determi- 
nant zero) we can take one of them constant but not zero, so that $\rho=2$; it then easily follows that

$$
\begin{gathered}
a_{11}=b_{11}, a_{22}=b_{22}, a_{33}=b_{33} / x_{2}^{2}, a_{23}=b_{23} / x_{2}, a_{31}=b_{31} / x_{2}, a_{12}=b_{12}, \\
a_{1}=b_{1} / x_{2}, a_{2}=b_{2} / x_{2}, a_{3}=b_{3} / x_{2}^{2}, a=b / x_{2}^{2},
\end{gathered}
$$

where we denote by the coefficients $b_{11} b_{22} \ldots b_{1} \ldots b$ functions which are independent both of $x_{1}$ and $x_{2}$.

\section{§ 5. Equations which admit the non-integrable group of the third order.}

We pass now to groups of the third order. It is known (LIE, vol. III, p. 716) that the only one which has not a pair of permutable transformations has the composition

$$
\left(X_{1} X_{2}\right)=X_{1}, \quad\left(X_{1} X_{3}\right)=2 X_{2}, \quad\left(X_{2} X_{3}\right)=X_{3}
$$

Taking

let

$$
X_{1} \equiv \frac{\partial}{\partial x_{1}}, \quad X_{2} \equiv x_{1} \frac{\partial}{\partial x_{1}}+x_{2} \frac{\partial}{\partial x_{2}}
$$

$$
X_{3} \equiv \xi_{1} \frac{\partial}{\partial x_{1}}+\xi_{2} \frac{\partial}{\partial x_{2}}+\xi_{3} \frac{\partial}{\partial x_{3}}+z \zeta \frac{\partial}{\partial z} .
$$

From the law of composition

$$
\begin{aligned}
& \frac{\partial}{\partial x_{1}} \xi_{1}=2 x_{1}, \quad \frac{\partial}{\partial x_{1}} \xi_{2}=2 x_{2}, \quad \frac{\partial}{\partial x_{1}} \xi_{3}=\frac{\partial}{\partial x_{1}} \zeta=0 ; \\
& X_{2} \xi_{1}=2 \xi_{1}, \quad X_{2} \xi_{2}=2 \xi_{2}, \quad X_{2} \xi_{3}=\xi_{3}, \quad X_{2} \zeta=\zeta .
\end{aligned}
$$

And we easily deduce

$$
\xi_{1}=x_{1}^{2}+x_{2}^{2} f_{1}\left(x_{3}\right), \quad \xi_{2}=2 x_{1} \dot{x}_{2}+x_{2}^{2} f_{2}\left(x_{3}\right), \quad \xi_{3}=x_{2} f_{3}\left(x_{3}\right), \quad \zeta=x_{2} f\left(x_{3}\right) .
$$

Now by any transformation of the form :

$$
x_{1}^{\prime}=x_{1}+x_{2} \phi_{1}\left(x_{3}\right), \quad x_{2}^{\prime}=x_{2} \phi_{2}\left(x_{3}\right), \quad x_{3}^{\prime}=\phi_{3}\left(x_{3}\right), \quad z^{\prime}=z \phi\left(x_{3}\right),
$$

the forms of $X_{1}$ and $X_{2}$ are unaltered.

Choose $\phi_{1}$ so that

$$
f_{1}+f_{2} \phi_{1}+f_{3} \frac{\partial \phi_{1}}{\partial x_{3}}=\phi_{1}^{2}
$$

If $f_{3}$ is not identically zero we can also choose $\phi_{2}$ and $\phi_{3}$ to satisfy the equations :

$$
f_{2} \phi_{2}+f_{3} \frac{\partial \phi_{2}}{\partial x_{3}}=2 \phi_{1} \phi_{2}, \quad f_{3} \frac{\partial \phi_{3}}{\partial x_{3}}=\phi_{2}
$$


finally choose $\phi$ so that

$$
f_{3} \frac{\partial \phi}{\partial x_{3}}+f \phi=0
$$

and we see that in the new variables $X_{1}, X_{2}$ are of the same form and $X_{3}$ is of the form :

$$
x_{1}^{2} \frac{\partial}{\partial x_{1}}+2 x_{1} x_{2} \frac{\partial}{\partial x_{2}}+x_{2} \frac{\partial}{\partial x_{3}} \text {. }
$$

If however $f_{3} \equiv 0$ it may be similarly seen by taking $\phi_{2}=f_{2}-2 \phi_{1}$, that $X_{3}$ can be thrown into the form :

$$
x_{1}^{2} \frac{\partial}{\partial x_{1}}+\left(2 x_{1} x_{2}+x_{2}^{2}\right) \frac{\partial}{\partial x_{2}}+x_{2} f\left(x_{3}\right) z \frac{\partial}{\partial z} .
$$

To show that this form cannot be admitted we take $\xi_{1}=x_{1}^{2}, \xi_{2}=2 x_{1} x_{2}+x_{2}^{2}$, $\xi_{3}=0$. Remembering that $a_{11}=b_{11}$, etc., and that $X=x_{1}^{2} \partial / \partial x_{1}+\left(2 x_{1} x_{2}\right.$ $\left.+x_{2}^{2}\right) \partial / \partial x_{2}$, we have $X a_{11}=X a_{22}=X a_{12}=0$, and

$$
X a_{13}=-\left(2 x_{1}+x_{2}\right) a_{13}, \quad X a_{23}=-\left(2 x_{1}+x_{2}\right) a_{23}, \quad X a_{33}=-2\left(2 x_{1}+x_{2}\right) a_{33}
$$

so that the equations (2) become

$$
\begin{array}{ll}
2 a_{11} x_{2}+2 a_{12}\left(2 x_{1}+x_{2}\right) & =\rho a_{12}, \\
2 a_{13} x_{1} & =\rho a_{13}-a_{13}\left(2 x_{1}+x_{2}\right), \\
2 a_{13} x_{2}+2 a_{23}\left(x_{1}+x_{2}\right) & =\rho a_{23}-a_{23}\left(2 x_{1}+x_{2}\right), \\
2 a_{11} x_{1} & =\rho a_{11}, \\
2 a_{21} x_{2}+2 a_{22}\left(x_{1}+x_{2}\right) & =\rho a_{22}, \\
0 & =\rho a_{33}-2 a_{33}\left(2 x_{1}+x_{2}\right) .
\end{array}
$$

Now if $a_{11} \neq 0$, one has $\rho=2 x_{1}, a_{33}=0$, and $a_{13}=0$; from which it follows that $a_{23}=0$, and the determinant reduces to zero. But if $a_{11}=0$ and $a_{12} \neq 0$, then $\rho=2\left(2 x_{1}+x_{2}\right)$, and by the fifth equation $a_{12} x_{2}=a_{22} x_{1}$; but by (8) $a_{12}$ and $a_{22}$ are independent of $x_{1}$ and $x_{2}$, and so $a_{12}=0$, which is contrary to the present hypothesis. Finally take $a_{11}=a_{12}=0$; if $a_{13}=0$, the determinant vanishes; but if $a_{13} \neq 0, \rho=4 x_{1}+x_{2}$, and $a_{22}=0$. In every case then the determinant vanishes, and we conclude that this type cannot be admitted.

Corresponding to the group of composition :

$$
\left(X_{1} X_{2}\right)=X_{1}, \quad\left(X_{1} X_{3}\right)=2 X_{2}, \quad\left(X_{2} X_{3}\right)=X_{3}
$$

we must then take as our type of infinitesimal transformation :

$$
X_{3} \equiv x_{1}^{2} \frac{\partial}{\partial x_{1}}+2 x_{1} x_{2} \frac{\partial}{\partial x_{2}}+x_{2} \frac{\partial}{\partial x_{3}} \text {. }
$$


We now have from (2)

$$
\begin{aligned}
& 4 a_{11} x_{1} \quad=X a_{11}+\rho a_{11}, \\
& 4 a_{12} x_{2}+4 a_{22} x_{1} \quad=X a_{22}+\rho a_{22} \text {, } \\
& 2 a_{23} \quad=X a_{33}+\rho a_{33} \text {, } \\
& 2\left(a_{13} x_{2}+a_{23} x_{1}\right)+a_{22}=X a_{23}+\rho a_{23} \text {, } \\
& 2 a_{31} x_{1}+a_{12}=X a_{13}+\rho a_{13} \text {, } \\
& 2\left(a_{11} x_{2}+a_{12} x_{1}\right)+2 a_{12} x_{1}=X a_{12}+\rho a_{12} \text {. }
\end{aligned}
$$

Unless $a_{11}=a_{22}=a_{12}=0$ (in which case the determinant would vanish) we see by taking $a_{11}=$ constant (or if this vanishes $a_{12}=$ constant, or if both vanish $a_{22}=$ constant) that $\rho=4 x_{1}$.

Also from (8)

$$
\begin{aligned}
X a_{11} & =x_{2} \frac{\partial}{\partial x_{3}} b_{11}, & X a_{22} & =x_{2} \frac{\partial}{\partial x_{3}} b_{22}, \\
X a_{33} & =-4 x_{1} a_{33}+x_{2}^{-1} \frac{\partial}{\partial x_{3}} b_{33}, & X a_{13} & =-2 x_{1} a_{13}+\frac{\partial}{\partial x_{3}} b_{13}, \\
X a_{23} & =-2 x_{1} a_{23}+\frac{\partial}{\partial x_{3}} b_{23}, & X a_{12} & =x_{2} \frac{\partial}{\partial x_{3}} b_{12} .
\end{aligned}
$$

Hence we deduce :

$$
2 a_{11}=\frac{\partial b_{12}}{\partial x_{3}}, \quad b_{12}=\frac{\partial b_{13}}{\partial x_{3}}, \quad 4 b_{12}=\frac{\partial b_{22}}{\partial x_{3}}, \quad b_{22}+2 b_{13}=\frac{\partial b_{23}}{\partial x_{3}}, \quad 2 b_{23}=\frac{\partial b_{33}}{\partial x_{3}} .
$$

Since $a_{11}$ is a constant we find the following solution of these equations :

$$
\begin{aligned}
& b_{11}=c_{11}, \\
& b_{12}=2 c_{11} x_{3}+c_{12}, \\
& b_{22}=4 c_{11} x_{3}^{2}+4 c_{12} x_{3}+c_{22}, \\
& b_{13}=c_{11} x_{3}^{2}+c_{12} x_{3}+c_{13}, \\
& b_{23}=2 c_{11} x_{3}^{3}+3 c_{12} x_{3}^{2}+\left(2 c_{13}+c_{22}\right) x_{3}+c_{23}, \\
& b_{33}=c_{11} x_{3}^{4}+2 c_{12} x_{3}^{3}+\left(2 c_{13}+c_{22}\right) x_{3}^{2}+2 c_{23} x_{3}+c_{33},
\end{aligned}
$$

where we denote by $c_{11}, c_{12} \cdots$ a system of constants.

The remaining equations (3) and (4) give after simple reduction :

and, therefore,

$$
b_{11}=\frac{\partial b_{1}}{\partial x_{3}}, \quad 2 b_{12}+2 b_{1}=\frac{\partial b_{2}}{\partial x_{3}}, \quad b_{2}=\frac{\partial b_{3}}{\partial x_{3}}, \quad 0=\frac{\partial b}{\partial x_{3}}
$$

$$
\begin{aligned}
& b_{1}=c_{11} x_{3}+c_{1}, \\
& b_{2}=3 c_{11} x_{3}^{2}+2\left(c_{12}+c_{1}\right) x_{3}+c_{2}, \\
& b_{3}=c_{11} x_{3}^{3}+\left(c_{12}+c_{1}\right) x_{3}^{2}+c_{2} x_{3}+c_{3}, \\
& b=c,
\end{aligned}
$$

where $c, c_{1}, c_{2}, c_{3}$ denote constants. 
§ 6. Equations admitting other groups of the third order.

The only other groups of the third order are (LIE, vol. III, p. 716) those which have the compositions :

$$
\left(X_{1} X_{2}\right)=0, \quad\left(X_{1} X_{3}\right)=X_{1}, \quad\left(X_{2} X_{3}\right)=m X_{2} \quad(m \text { constant }) ;
$$

$$
\left(X_{1} X_{2}\right)=0, \quad\left(X_{1} X_{3}\right)=X_{1}, \quad\left(X_{2} X_{3}\right)=X_{1}+X_{2} \text {; }
$$

$$
\left(X_{1} X_{2}\right)=0, \quad\left(X_{1} X_{3}\right)=0, \quad\left(X_{2} X_{3}\right)=X_{1}
$$

$$
\left(X_{1} X_{2}\right)=0, \quad\left(X_{2} X_{3}\right)=0 \quad, \quad\left(X_{1} X_{3}\right)=0 \text {. }
$$

Taking (II) first we have seen that we may take $X_{1} \equiv \partial_{/} \partial x_{1}, X_{2} \equiv \partial / \partial x_{2}$; if $X_{3} \equiv \xi_{1} \partial / \partial x_{1}+\xi_{2} \partial / \partial x_{2}+\xi_{1} \partial / \partial x_{3}+\xi z \partial / \partial z$, we have from the composition of the group :

$$
\xi_{1}=x_{1}+f_{1}\left(x_{3}\right), \quad \xi_{2}=m x_{2}+f_{2}\left(x_{3}\right), \quad \xi_{3}=f_{3}\left(x_{3}\right), \quad \zeta=f\left(x_{3}\right) .
$$

Noticing that $X_{1}$ and $X_{2}$ are unaltered in form by any transformation :

$$
x_{1}^{\prime}=x_{1}+\phi_{1}\left(x_{3}\right), \quad x_{2}^{\prime}=x_{2}+\phi_{2}\left(x_{3}\right), \quad x_{3}^{\prime}=\phi_{3}\left(x_{3}\right), \quad z^{\prime}=z \phi\left(x_{3}\right),
$$

we see that by properly choosing the forms $\phi_{1}, \phi_{2}, \phi_{3}, \phi$, we can without altering the forms of $X_{1}$ and $X_{2}$ throw $X_{3}$ into one of the forms:

$$
\begin{gathered}
x_{1} \frac{\partial}{\partial x_{1}}+m x_{2} \frac{\partial}{\partial x_{2}}+\frac{\partial}{\partial x_{3}} ; \\
x_{1} \frac{\partial}{\partial x_{1}}+m x_{2} \frac{\partial}{\partial x_{2}}+f\left(x_{3}\right) z \frac{\partial}{\partial z} .
\end{gathered}
$$

In (a), remembering that all the coefficients $a_{h \kappa}, a_{h}, a$ now depend only on $x_{3}$, we see that $(2),(3), 4$ become :

$$
\left\{\begin{array}{rlrl}
2 a_{11} & =\rho a_{11}+\frac{\partial a_{11}}{\partial x_{3}}, & 2 m a_{22} & =\rho a_{22}+\frac{\partial a_{22}}{\partial x_{3}} \\
0 & =\rho a_{33}+\frac{\partial a_{33}}{\partial x_{3}}, & m a_{23} & =\rho a_{23}+\frac{\partial a_{23}}{\partial x_{3}} \\
a_{31} & =\rho a_{13}+\frac{\partial a_{13}}{\partial x_{3}}, & (m+1) a_{12} & =\rho a_{12}+\frac{\partial a_{12}}{\partial x_{3}} \\
a_{1} & =\rho a_{1}+\frac{\partial a_{1}}{\partial x_{3}}, \\
0 & =\rho a_{3}+\frac{\partial a_{3}}{\partial x_{3}}, & m a_{2} & =\rho a_{2}+\frac{\partial a_{2}}{\partial x_{3}} \\
& 0 & =\rho a+\frac{\partial a}{\partial x_{3}}
\end{array}\right.
$$


Now first suppose that $a_{33}$ is not zero; then we take it a constant $c_{33}$ and we get the solution of (9) given in the first column of the following table :

$$
\begin{array}{l|l|l}
a_{33}=c_{33} & a_{33}=0 & a_{33}=0 \\
a_{11}=c_{11} e^{2 x_{3}} & a_{11}=c_{11} e^{(2-m) x_{3}} & a_{11}=c_{11} e^{(2-2 m) x_{3}} \\
a_{22}=c_{22} e^{2 m x_{3}} & a_{22}=c_{22} e^{m x_{3}} & a_{22}=a_{22} \\
a_{23}=c_{23} e^{m x_{3}} & a_{23}=c_{23} & a_{23}=0 \\
a_{31}=c_{31} e^{x_{3}} & a_{31}=c_{31} e^{(1-m) x_{3}} & a_{31}=c_{13} e^{(1-2 m) x_{3}} \\
a_{12}=c_{12} e^{(1+m) x_{3}} & a_{12}=c_{12} e^{x_{3}} & a_{12}=c_{12} e^{(1-m) x_{3}} \\
a_{1}=c_{1} e^{x_{3}} & a_{1}=c_{1} e^{(1-m) x_{3}} & a_{1}=c_{1} e^{(1-2 m) x_{9}} \\
a_{2}=c_{2} e^{m x_{3}} & a_{2}=c_{2} & a_{2}=c_{2} e^{-m x_{3}} \\
a_{3}=c_{3} & a_{3}=c_{3} e^{-m x_{3}} & a_{3}=c_{3} e^{-2 m x_{3}} \\
a=c & a=c e^{-m x_{3}} & a=c e^{-2 m x_{3}} \\
\rho=0 & \rho=m & \rho=2 m
\end{array}
$$

where we always use the letters $c_{11} c_{12} \cdots c_{1} \cdots c$ to denote constants.

If $a_{33}=0$ but $a_{23} \neq 0$ then we obtain the solution given in the second column. If $a_{33}=a_{23}=0$ then we cannot have $a_{22}=0$ (since then the determinant would vanish) and the only other solution is given in the third column. Notice that of these three solutions the second and the third are really included under the first and do not give distinct forms of (1).

Take now the case when $X_{3}$ is of type $(\beta)$; we have

$$
\begin{aligned}
\rho a_{33} & =0, & \rho a_{23} & =m a_{23}, \\
\rho a_{13} & =a_{13}, & \rho a_{22} & =2 m a_{22}, \\
\rho a_{11} & =2 a_{11}, & \rho a_{12} & =(1+m) a_{12}, \\
\rho a_{1} & =a_{1}-a_{13} \frac{\partial f}{\partial x_{3}}, & \rho a_{2} & =m a_{2}-a_{23} \frac{\partial f}{\partial x_{3}}, \\
\rho a_{3} & =-a_{33} \frac{\partial f}{\partial x_{3}}, & \rho a & =-2 a_{3} \frac{\partial f}{\partial x_{3}}-a_{33} \frac{\partial^{2} f}{\partial x_{3}^{2}} .
\end{aligned}
$$

And it is not difficult to see that the only solutions which do not cause the determinant to vanish are the set given in the table of $\$ 7$ under $\operatorname{II}(\beta)$. Notice that the general form of $\operatorname{II}(\beta)$ is not admitted, but only the cases when $f\left(x_{3}\right)$ is a constant, and $m=-1,2$, or $\frac{1}{2}$. 
§ 7. Remaining groups of the third order and general conclusions.

We may similarly prove hat the groups (III) can be thrown into one of the two forms :

$$
\begin{aligned}
& \text { (a) } X_{1}=\frac{\partial}{\partial x_{1}}, \quad X_{2}=\frac{\partial}{\partial x_{2}}, \quad X_{3}=\left(x_{1}+x_{2}\right) \frac{\partial}{\partial x_{1}}+x_{2} \frac{\partial}{\partial x_{2}}+\frac{\partial}{\partial x_{3}} ; \\
& \text { ( } \beta) \quad X_{1}=\frac{\partial}{\partial x_{1}}, \quad X_{2}=\frac{\partial}{\partial x_{2}}, \quad X_{3}=\left(x_{1}+x_{2}\right) \frac{\partial}{\partial x_{1}}+x_{2} \frac{\partial}{\partial x_{2}}+f\left(x_{3}\right) z \frac{\partial}{\partial z} .
\end{aligned}
$$

And $t$ may be shown by methods similar to those, already employed that the latter form cannot be admitted by a differential equation with non-vanishing determinant.

The first gives :

$$
\begin{aligned}
2\left(a_{11}+a_{12}\right) & =\rho a_{11}+\frac{\partial a_{11}}{\partial x_{3}}, & 2 a_{22} & =\rho a_{22}+\frac{\partial a_{22}}{\partial x_{3}}, \\
0 & =\rho a_{33}+\frac{\partial a_{33}}{\partial x_{3}}, & 2 a_{12}+a_{22} & =\rho a_{12}+\frac{\partial a_{12}}{\partial a_{3}}, \\
a_{13}+a_{23} & =\rho a_{13}+\frac{\partial a_{13}}{\partial x_{3}}, & a_{23} & =\rho a_{23}+\frac{\partial a_{23}}{\partial x_{3}}, \\
a_{1}+a_{2} & =\rho a_{1}+\frac{\partial a_{1}}{\partial x_{3}}, & a_{2} & =\rho a_{2}+\frac{\partial a_{2}}{\partial x_{3}}, \\
0 & =\rho a_{3}+\frac{\partial a_{3}}{\partial x_{3}}, & 0 & =\rho a+\frac{\partial a}{\partial x_{3}}
\end{aligned}
$$

And of these equations we have the solution $\operatorname{III}(a)$ of the table.

The group (IV) has two possible types:

$$
\begin{gathered}
X_{1} \equiv \frac{\partial}{\partial x_{1}}, \quad X_{2}=\frac{\partial}{\partial x_{2}}, \quad X_{3}=x_{2} \frac{\partial}{\partial x_{1}}+\frac{\partial}{\partial x_{3}} \\
X_{1} \equiv \frac{\partial}{\partial x_{1}}, \quad X_{2}=\frac{\partial}{\partial x_{2}}, \quad X_{3}=x_{2} \frac{\partial}{\partial x_{1}}+x_{3} \frac{\partial}{\partial x_{2}}+f\left(x_{3}\right) z \frac{\partial}{\partial z} .
\end{gathered}
$$

Type (a) leads to

$$
\begin{aligned}
\rho a_{33}+\frac{\partial a_{33}}{\partial x_{3}} & =0, & \rho a_{22}+\frac{\partial a_{22}}{\partial x_{3}} & =0, \\
\rho a_{23}+\frac{\partial a_{23}}{\partial x_{3}} & =0, & \rho a_{13}+\frac{\partial a_{13}}{\partial x_{3}} & =a_{23}, \\
\rho a_{12}+\frac{\partial a_{12}}{\partial x_{3}} & =a_{22}, & \rho a_{11}+\frac{\partial a_{11}}{\partial x_{3}} & =2 a_{12}, \\
\frac{\partial a_{1}}{\partial x_{3}} & =a_{2}, & \frac{\partial a_{2}}{\partial x_{3}} & =0, \\
\frac{\partial a_{3}}{\partial x_{3}} & =0, & \frac{\partial a}{\partial x_{3}} & =0 .
\end{aligned}
$$


Of these the most general solution of non-vanishing determinant is found under $\operatorname{IV}(a)$.

Type $(\beta)$ leads to

$$
\begin{aligned}
\rho a_{33} & =0, & \rho a_{23} & =a_{33}, \\
\rho a_{22} & =2 a_{23}, & \rho a_{13} & =a_{23}, \\
\rho a_{12} & =a_{22}+a_{13}, & \rho a_{11} & =2 a_{12}, \\
a_{2} & =a_{13} \frac{\partial f}{\partial x_{3}}+\rho a_{1}, & a_{3} & =a_{23} \frac{\partial f}{\partial x_{3}}+\rho a_{2}, \\
0 & =a_{33} \frac{\partial f}{\partial x_{3}}+\rho a_{33}, & 0 & =a_{33} \frac{\partial^{2} f}{\partial x_{3}^{2}}+2 a_{3} \frac{\partial f}{\partial x_{3}}+\rho a,
\end{aligned}
$$

of which the solution is given under $\operatorname{IV}(\beta)$.

Finally the only type of group (V) which could be admitted is $X_{1}=\partial / \partial x_{1}$, $X_{2}=\partial / \partial x_{2}, X_{3}=\partial / \partial x_{3}$. We see this by noticing that when we bring $X_{1}$ and $X_{2}$ into the forms $\partial / \partial x_{1}$ and $\partial / \partial x_{2}$ respectively, then $X_{3}$ is from the laws of composition of the form :

$$
f_{1}\left(x_{3}\right) \frac{\partial}{\partial x_{1}}+f_{2}\left(x_{3}\right) \frac{\partial}{\partial x_{2}}+f_{3}\left(x_{3}\right) \frac{\partial}{\partial x_{3}}+f\left(x_{3}\right) z \frac{\partial}{\partial z} .
$$

If $f_{3}\left(x_{3}\right) \equiv 0$, it may easily be verified, by taking $x_{3}^{\prime}=f_{1}\left(x_{3}\right)$ and throwing $X_{3}$ into the form $x_{3} \partial / \partial x_{1}+f_{2}\left(x_{3}\right) \partial / \partial x_{2}+f\left(x_{3}\right) z \partial / \partial z$, that the group cannot be admitted without causing the determinant to vanish.

If $f_{3}\left(x_{3}\right) \neq 0$, by a transformation of the form :

$$
x_{1}^{\prime}=x_{1}+\phi_{1}\left(x_{3}\right), \quad x_{2}^{\prime}=x_{2}+\phi_{2}\left(x_{3}\right), \quad x_{3}^{\prime}=\phi_{3}\left(x_{3}\right), \quad z^{\prime}=z \phi\left(x_{3}\right),
$$

we can, without altering the forms of $X_{1}$ and $X_{2}$, throw $X_{3}$ into the form $\partial / \partial x_{3}$.

The necessary and sufficient condition that (1) should admit such a group is then that by point-transformation of the form (7) it can be thrown into the form of a linear partial differential equation with constant coefficients.

We can now tabulate the results at which we have arrived in this paper. If the equation :

$$
\begin{aligned}
a_{11} z_{11}+a_{22} z_{22}+ & a_{33} z_{33}+2 a_{23} z_{23}+2 a_{31} z_{31}+2 a_{12} z_{12}+2 a_{1} z_{1} \\
& +2 a_{2} z_{2}+2 a_{3} z_{3}+a z=0
\end{aligned}
$$

of non-vanishing determinant, admits at least one infinitesimal transformation, it can be reduced by point transformation to such a form that all the coefficients are independent of the variable $x_{1}$. If it admits at least two such transformations it can be reduced to one of two forms, the first form being that in which the coefficients are independent of $x_{1}$ and $x_{2}$, and the second that in which

$$
\begin{gathered}
a_{11}=b_{11}, \quad a_{22}=b_{22}, \quad a_{33}=b_{33} / x_{2}^{2}, \quad a_{23}=b_{23} / x_{2}, \quad a_{31}=b_{31} / x_{2}, \quad a_{12}=b_{12}, \\
a_{1}=b_{1} / x_{2}, \quad a_{2}=b_{2} / x_{2}, \quad a_{3}=b_{3} / x_{2}^{2}, \quad a=b / x_{2}^{2}
\end{gathered}
$$

where all the quantities $b_{i k}, b_{i}, b$ are independent of $x_{1}$ and $x_{2}$. 
An equation which admits at least three infinitesimal transformations can be reduced to some one of the forms given in the accompanying table, where the number at the head of a column indicates the group corresponding to the equation given in that column, and where the $b_{i k}, b_{i}, b$ are independent of $x_{1}$ and $x_{2}$, and the $c_{i k}, c_{i}, c$ are constants. I hope in some future paper to discuss the further relations necessary between the constants $c_{i k} \cdots$ and the functions $b_{i k} \cdots$ in order that the equation may admit groups of order above the third.

\begin{tabular}{|c|c|c|c|c|c|}
\hline Coeffic & lent of & $\operatorname{II}(a)$ & $\mathbf{I I}(\boldsymbol{\beta})$ & $\mathbf{I I}(\boldsymbol{\beta})$ & $\mathbf{I I}(\boldsymbol{\beta})$ \\
\hline$z_{11}$ & $c_{11}$ & $c_{11} e^{2 x_{3}}$ & 0 & $b_{11}$ & 0 \\
\hline$z_{22}$ & $4 c_{11} x_{3}^{2}+4 c_{12} x_{3}+c_{22}$ & $c_{22} e^{2 m x_{3}}$ & 0 & 0 & $b_{22}$ \\
\hline$z_{33}$ & $\left\{c_{11} x_{3}^{4}+2 c_{12} x_{3}^{3}+\left(c_{22}+2 c_{13}\right) x_{3}^{2}+2 c_{23} x_{3}+c_{33}\right\} \div x_{2}^{2}$ & $c_{33}$ & $\overline{b_{33}}$ & 0 & 0 \\
\hline $2 z_{23}$ & $\left\{2 c_{11} x_{3}^{3}+3 c_{12} x_{3}^{2}+\left(c_{22}+2 c_{13}\right) x_{3}+c_{23}\right\} \div x_{2}$ & $c_{23} e^{m x_{3}}$ & 0 & $\overline{b_{23}}$ & 0 \\
\hline $2 z_{31}$ & $\left(c_{11} x_{3}^{2}+c_{12} x_{3}+c_{13}\right) \div x_{2}$ & $c_{31} e^{x_{3}}$ & 0 & 0 & $b_{13}$ \\
\hline $2 z_{12}$ & $2 \overbrace{11} x_{3}+c_{12}$ & $c_{12} e^{(1+m) x_{3}}$ & $\overline{b_{12}}$ & 0 & 0 \\
\hline $2 z_{1}$ & $\left(c_{11} x_{3}+c_{1}\right) \div x_{2}$ & $c_{1} e^{x_{3}}$ & 0 & 0 & $b_{1}$ \\
\hline $2 z_{2}$ & $\left\{3 c_{11} x_{3}^{2}+2\left(c_{12}+c_{1}\right) x_{3}+c_{2}\right\} \div x_{2}$ & $c_{2} e^{m x_{2}}$ & 0 & $b_{2}$ & 0 \\
\hline $2 z_{3}$ & $\left\{2 c_{11} x_{3}^{3}+2\left(c_{12}+c_{1}\right) x_{3}^{2}+2 c_{2} x_{3}+c_{3}\right\} \div x_{2}^{2}$ & $c_{3}$ & $b_{3}$ & 0 & 0 \\
\hline $\bar{z}$ & $c \div x_{2}^{2}$ & $c$ & $b$ & 0 & 0 \\
\hline
\end{tabular}

\begin{tabular}{|c|c|c|c|c|}
\hline Coeffic & $\operatorname{III}(a)$ & $\operatorname{IV}(a)$ & $\operatorname{IV}(\beta)$ & $\mathrm{v}$ \\
\hline$z_{11}$ & $\left(c_{22} x_{3}^{2}+2 c_{12} x_{3}+c_{11}\right) e^{2 x_{3}}$ & $c_{22} x_{3}^{2}+2 c_{12} x_{3}+c_{11}$ & $b_{11}$ & $c_{11}$ \\
\hline$z_{22}$ & $c_{22} e^{2 x_{3}}$ & $c_{22}$ & $b_{22}$ & $c_{22}$ \\
\hline$z_{33}$ & $c_{33}$ & $c_{33}$ & 0 & $c_{33}$ \\
\hline $2 z_{23}$ & $c_{23} e^{x_{3}}$ & $c_{23}$ & 0 & $c_{23}$ \\
\hline$\overline{2 z_{31}}$ & $\left(c_{23} x_{3}+c_{13}\right) e^{x_{3}}$ & $c_{23} x_{3}+c_{13}$ & $-b_{22}$ & $c_{31}$ \\
\hline $2 z_{12}$ & $\left(c_{22} x_{3}+c_{12}\right) e^{2 x_{3}}$ & $c_{22} x_{3}+c_{12}$ & 0 & $c_{12}$ \\
\hline$\overline{2 z_{1}}$ & $\left(c_{2} x_{3}+c_{1}\right) e^{x_{3}}$ & $c_{2} x_{3}+c_{1}$ & $b_{1}$ & $c_{1}$ \\
\hline $2 z_{2}$ & $c_{2} e^{x_{3}}$ & $c_{2}$ & $-b_{22} \frac{\partial f\left(x_{3}\right)}{\partial x_{3}}$ & $c_{2}$ \\
\hline$\overline{2 z_{3}}$ & $c_{3}$ & $c_{3}$ & 0 & $c_{3}$ \\
\hline$z$ & $c$ & $c$ & $b$ & $c$ \\
\hline
\end{tabular}

HeRTFORd COLLEGE, OXFORD,

December 7, 1899. 